\title{
Steady states of max-Lukasiewicz fuzzy systems
}

\author{
Zuzana Němcová ${ }^{1}$ \\ ${ }^{1}$ University of Hradec Králové, Hradec Králové, Czech Republic
}

\begin{abstract}
The paper gives the systematic characterization of eigenspace in $\max -T$ algebra where $\mathrm{T}$ is equal to Łukasiewicz $t$-norm. Max-Łukasiewicz fuzzy algebra can be used for the description of the states of Discrete-event systems. The states can represent a balance of the resource unit expended during the evolution of the system (for example fuel or money). In the contribution, the classification of max-Łukasiewicz eigenspaces is described and explained by the two-dimensional examples - in this case it is possible to accompany the example with illustrative graphs. However the description of the eigenspace for the higher dimensions is also outlined.
\end{abstract}

Keywords: Fuzzy algebra, $t$-norms, maxŁukasiewicz algebra, steady states

\section{Introduction}

Extremal algebras are used mainly for describing and studying systems working in discrete time. During the operation of such systems the steady state can arise. The system is described by transition matrix and the eigenvector(s) of such matrix represents the steady states of the system.

Max-T fuzzy algebra is defined over the interval $[0,1]$ and uses instead of conventional operations of addition and multiplication the operations of maximum and one of the triangular norms, the so-called $t$-norms. These operations are extended in a natural way to cartesian products of vectors and matrices. $T$-norms were introduced by Schweizer and Sklar in [8] in the context of probabilistic metric spaces. $T$ norms are used to interpret the conjunction in the fuzzy logics and intersection of fuzzy sets. These functions have applications in many areas such as decision making processes, statistics and game theory, information and data fusion, probability theory and risk management. The $t$-norms together with $t$-conorms play the key role in fuzzy set theory.

The fuzzy extension of description logics, the formalism for the representation of structured knowledge used frequently in the design of ontologies, is described in [1]. The meaning of ontologies has increased in the last decades. Ontologies have been successfully used as part of expert and multiagent systems, as a knowledge base in robotics as well as the core element in the Semantic Web (aims at converting the current web into a "web of data" by defining the meaning of information).
Although there exist many $T$-norm families (for example, the Aczel-Alsina, the Jane Doe1, Hamacher, Einstein product - for the overview see [2]), let us mention four main $t$-norms - Łukasiewicz, Gödel, Product and Drastic.

The Łukasiewicz norm might be considered as a logic of absolute or metric comparison. The Łukasiewicz conjunction is computed as

$$
x \otimes_{L} y=\max \{x+y-1,0\} .
$$

Gödel norm is the simplest norm; the conjunction is defined as a minimum of the entries - of the truth degrees of the constituents. Gödel logic is logic with a relative comparison.

$$
x \otimes_{G} y=\min (x, y)
$$

The definition of the product norm follows:

$$
x \otimes_{p} y=x \cdot y .
$$

The drastic triangular norm (in literature, there can be also found the term "weakest norm") is basic example of a non-divisible $t$-norm on any partially ordered set. The drastic triangular norm is defined as follows:

$$
x \otimes_{\mathrm{d}} y=\left\{\begin{array}{cl}
\min (x, y) & \text { if } \max (x, y)=1 \\
0 & \text { if } \max (x, y)<1
\end{array}\right.
$$

The eigenvalues and eigenvectors belong to important characteristics of the system described by these fuzzy algebras. For the case of drastic and product $t$-norm, the eigenspace structure have been already studied. The paper [4] published in the journal Fuzzy Sets and Systems deals with maxdrast algebra. The investigation of the eigenspace in max-prod algebra [7] is in preparation. Finally, papers $[6,3]$ deals with Eukasiewicz fuzzy algebra.

The state of the system in time $t$ can be described by the state vector, say $x(t)$. The transition matrix, denoted by $A$, describes the transitions of the system from one state to another. By the multiplication of transition matrix and the state vector, the next state of the system, $x(t+1)$, is obtained; it can be written $A \otimes x(t)=x(t+1)$. During the operation of the system, after some time, it can happen that the system reaches a steady state. In max-€ukasiewicz fuzzy algebra the state vectors of steady states correspond to the eigenvectors of the transition matrix $A$.

Łukasiewicz arithmetical conjunction can be used in many types of situations. The fact that the number 1 is subtracted from the sum of the components 
and the maximum with zero is taken, leads to the observation that the count of the operation is the remainder, some part that is over the unit. Following this idea, the conjunction can be used to compute, for example, the amount of money that should be paid off for the phone bill where $x$ can be the price for the SMS in total and $y$ can represent the price for the calls in total. Number 1 here is substituted with the amount of lump sum. There are other similar situations, for example, the data backup on the server, maximal capacity of the pond, overdraft of the project funds, or savings of the partners with a common bill.

\section{Eigenproblem in Max-Łukasiewicz fuzzy algebra}

Max-Łukasiewicz algebra uses, as was written above, two binary operations - the operation of addition $x \oplus y=\max (x, y)$ and the operation of multiplication $x \otimes_{L} y=\max \{x+y-1,0\}$. The operation $\otimes_{L}$ has, as in conventional algebra, the priority over the operation $\oplus$. These operations are formally extended to matrices and vectors, again, similarly as in linear algebra. That is if matrices $A=\left(a_{i j}\right)$ and $B=\left(b_{i j}\right)$ are of compatible sizes then it can be written $A \oplus B=a_{i j} \oplus b_{i j}=\max \left(a_{i j}, b_{i j}\right) ; A \otimes_{L}$ $B=\bigoplus_{k} a_{i k} \otimes_{L} b_{k j}=\max _{k}\left(\max \left(a_{i j}+b_{i j}-1,0\right)\right)$; $\alpha \otimes_{L} A=A \otimes_{L} \alpha=\alpha \otimes_{L} a_{i j}$ for $\alpha \in \mathcal{R}$.

To solve the eigenproblem in max-Łukasiewicz algebra means to find such nontrivial vector $x$ (called the eigenvector) for some $\lambda$ (called the eigenvalue) such that it holds

$$
A \otimes_{L} x=\lambda \otimes_{L} x .
$$

This equation can be converted into the language of the so-called tropical linear algebra, i.e. max-plus algebra, because, as we will see, these two structures are closely related. The max-plus algebra is denoted by $\mathcal{R}_{\max }=(\bar{R}, \oplus, \otimes, \varepsilon, e)$, where $\bar{R}$ is the set of real numbers extended by the infinite value $\varepsilon=-\infty$ and zero element $e=0$. Binary operations $\oplus, \otimes$ are defined on $\bar{R}$ as $a \oplus b=\max (a, b)$ and $a \otimes b=a+b$.

The developed theory of well known max-plus algebra can be applied in a following way - let's have a closer look at the Łukasiewicz conjunction and rewrite the equation using max-plus operators:

$$
x \otimes_{L} y=(x-1) \otimes y \oplus 0
$$

Then the equation (5) of the eigenproblem in maxŁukasiewicz algebra can be converted into the maxplus one in the following way:

$$
A^{(1)} \otimes x \oplus \mathbf{0}=(\lambda-1) \otimes x \oplus \mathbf{0},
$$

where $A^{(1)}$ is the matrix with all entries equal to $\left(a_{i j}-1\right)$ and $\mathbf{0}$ is a vector with zero entries.

Some examples with illustrative graphs and pictures are given for two- and three-dimensional matrix. For two dimensions the eigenspaces can be clearly depicted without the need to have an interactive environment as it would be in case of four+ dimensions. To generate some graphical representation for higher dimensions is unfortunately not clearly arranged, however the computation of the characteristics is more complex, but analogical, and will be also shown in further text.

\section{Characterization of the eigenspace for two-dimensional matrix}

Let us consider the two-dimensional matrix $A$, vector $x$ and the eigenvalue $\lambda$. The eigenproblem can be written as:

$$
\left(\begin{array}{ll}
a_{11} & a_{12} \\
a_{21} & a_{22}
\end{array}\right) \otimes_{L}\left(\begin{array}{l}
x_{1} \\
x_{2}
\end{array}\right)=\lambda \otimes_{L}\left(\begin{array}{l}
x_{1} \\
x_{2}
\end{array}\right),
$$

and converted to tropical algebra

$$
\begin{array}{r}
\left(\begin{array}{ll}
a_{11}-1 & a_{12}-1 \\
a_{21}-1 & a_{22}-1
\end{array}\right) \otimes\left(\begin{array}{l}
x_{1} \\
x_{2}
\end{array}\right) \oplus\left(\begin{array}{l}
0 \\
0
\end{array}\right)= \\
(\lambda-1) \otimes\left(\begin{array}{l}
x_{1} \\
x_{2}
\end{array}\right) \oplus\left(\begin{array}{l}
0 \\
0
\end{array}\right) .
\end{array}
$$

To solve the eigenproblem, two equations can be identified and solved. The first equation is of form:

$\left(a_{11}+x_{1}-1\right) \vee 0 \vee\left(a_{12}+x_{2}-1\right) \vee 0=\left(\lambda+x_{1}-1\right) \vee 0$

For simplification of the equation and preparation for further use, the element $(1-\lambda)$ can be added to both sides of the equation (with an effect of the variables separation on the right side of the equation):

$$
\begin{array}{r}
\left(a_{11}+x_{1}-\lambda\right) \vee(1-\lambda) \vee\left(a_{12}+x_{2}-\lambda\right) \vee \\
(1-\lambda)=x_{1} \vee(1-\lambda)
\end{array}
$$

Proposition 3.1 For $x_{1} \in(1-\lambda, 1\rangle$, the equation (11) holds if and only if either

$$
\begin{aligned}
& a_{11}=\lambda \wedge a_{12}-\lambda+x_{2} \leq x_{1} \\
& \text { or } \\
& a_{11} \leq \lambda \wedge a_{12}-\lambda+x_{2}=x_{1}
\end{aligned}
$$

Proof: It is easily seen that for the case $(1-\lambda)<$ $x_{1} \leq 1$ it holds $\left(a_{11}+x_{1}-\lambda\right) \vee(1-\lambda) \vee\left(a_{12}+x_{2}-\right.$ ג) $\vee(1-\lambda)=x_{1}$. The equation is satisfied if and only if $\left(a_{11}+x_{1}-\lambda\right)=x_{1}$ and other terms of the equation are less or equal $x_{1}$, or $\left(a_{12}+x_{2}-\lambda\right)=x_{1}$ and other terms are less or equal $x_{1}$.

Proposition 3.2 For $x_{1} \in\langle 0,1-\lambda\rangle$, the equation (11) holds if and only if the condition

$$
x_{1} \leq 1-a_{11} \wedge x_{2} \leq 1-a_{12}
$$

is satisfied. 
PROOF: In the case $0<x_{1} \leq(1-\lambda)$ it holds $\left(a_{11}+x_{1}-\lambda\right) \vee(1-\lambda) \vee\left(a_{12}+x_{2}-\lambda\right) \vee(1-\lambda)=$ $(1-\lambda)$ and this is true if and only if both terms $\left(a_{11}+x_{1}-\lambda\right)$ and $\left(a_{12}+x_{2}-\lambda\right)$ are less or equal $(1-\lambda)$.

Similarly for the second equation it holds

$$
\begin{array}{r}
\left(a_{21}+x_{1}-\lambda\right) \vee(1-\lambda) \vee\left(a_{22}+x_{2}-\lambda\right) \vee \\
(1-\lambda)=x_{2} \vee(1-\lambda)
\end{array}
$$

Proposition 3.3 For $x_{2} \in(1-\lambda, 1\rangle$, the equation (15) holds if and only if either

$$
\begin{aligned}
& a_{22}=\lambda \wedge a_{21}-\lambda+x_{1} \leq x_{2} \\
& \quad \text { or } \\
& a_{22} \leq \lambda \wedge a_{21}-\lambda+x_{1}=x_{2} .
\end{aligned}
$$

Proof: Again, for the case $(1-\lambda)<x_{2} \leq 1$ the equation (15) is true if and only if $\left(a_{21}+x_{1}-\lambda\right)=x_{2}$ and other terms of the equation are less or equal $x_{2}$, or $\left(a_{22}+x_{2}-\lambda\right)=x_{2}$ and other terms are less or equal $x_{2}$.

Proposition 3.4 For $x_{2} \in\langle 0,1-\lambda\rangle$, the equation (11) holds if and only if the condition

$$
x_{2} \leq 1-a_{22} \wedge x_{1} \leq 1-a_{21} .
$$

is satisfied.

Proof: For the case $0<x_{2} \leq(1-\lambda)$ it holds that both terms, $\left(a_{21}+x_{1}-\lambda\right)$ and $\left(a_{22}+x_{2}-\lambda\right)$ have to be less or equal $(1-\lambda)$.

The solution set of (8) is tropically convex. We can say that it is the tropical convex hull defined by the finite number of points. That is, if the set contains points $x, y$ then the tropical segment joining $x$ and $y$ also belongs to the set. Examples of tropical segments between three points are shown in the left part of Figure 1, tropical segment is a combination of the vertical, horizontal and diagonal segments, for details see [5]. The right part of figure depicts the tropical convex hull defined by three points.
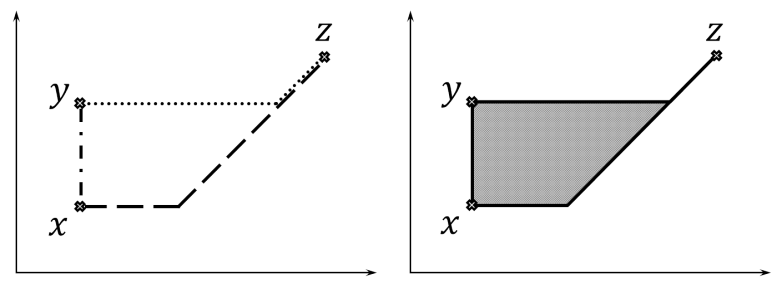

Figure 1: Tropical segments and tropical convex hull for the case of three points $x, y, z$.

Now we can picture the solution set for the first equation (11) using some of the above written conditions. Let's say for the entries $a_{11}<a_{12}<\lambda$ we have to consider the conditions (13) and (14). Figure 2 shows the solution set. For the cases where $x_{1}>(1-\lambda)$ it holds that $a_{12}-\lambda+x_{2}=x_{1}$. Because in this case $a_{12}-\lambda<0$, the solution of the equation is the straight segment above the diagonal. For the cases where $x_{1} \leq(1-\lambda)$ the condition (14) is considered. The solution set of the first equation is then the union of solutions of two above mentioned domains.

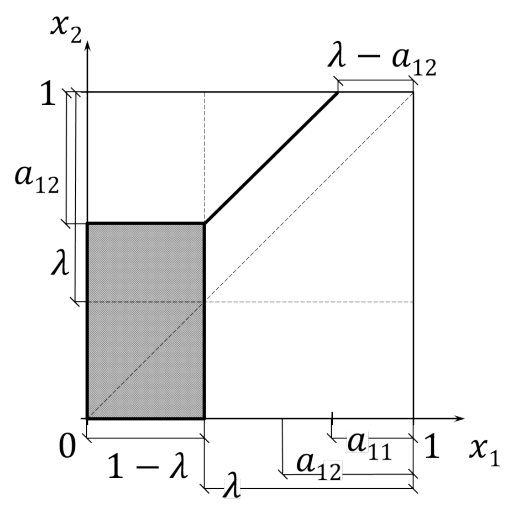

Figure 2: The solution set of the first equation in case $a_{11}<a_{12}<\lambda$

Along the same lines it is possible to construct the solution set for other possible positions of the parameter $\lambda$ and the variables from the equation. Although there are six possibilities to place $\lambda$, three special types of shapes of solution sets for each of equations can be observed. These types depend on the relative positions of $\lambda$ and the diagonal entries of the matrix. For the two-diagonal example, we can also say that the entries of the first column of $A$ have an influence on the height of the solution set, whereas the entries of the second column influence the width of the solution set of particular equation.

Figure 3 shows all three types of solution sets for each equation. The numbers in the notation stand for the particular equation (whether it is the solution set for the first or the second equation) and the Greek letter means the type. The types are generally influenced by the position of $\lambda$ and the diagonal entry of the matrix. For the $i$ th equation it holds that the solution set is of type

- $\alpha$ if and only if $a_{i i}<\lambda$

- $\beta$ if and only if $a_{i i}=\lambda$

- $\gamma$ if and only if $a_{i i}>\lambda$

Note that the values of particular entries are denoted in the picture by a complement to number one, for example, $\bar{\lambda}=1-\lambda$. The possible changes in the shape in dependence on the size of $a_{i j}$ are also outlined.

The solution sets denoted as $1 \alpha$ and $2 \alpha$ are similar each other by the shape of the solution set - a rectangle connected with a segment of solutions in the upper right corner. In case of the type $1 \alpha$ the 
parameter $1-a_{12}$ influences the height of the rectangle and the value $1-\lambda$ influences the width. The limits for the case $2 \alpha$ are symmetric - the parameter $1-a_{21}$ influences the width and $1-\lambda$ has impact on the height of the solution set.

In case of type $\beta$ the width of solution set $1 \beta$ in the picture (height for $2 \beta$ ) is maximal possible and the height is influenced by the value $1-a_{12}\left(1-a_{21}\right.$ for $2 \beta$ ) and the point of intersection with $\lambda$.

It can be seen from the picture that the third type, $\gamma$, is the simplest one. The value $1-a_{12}$ influences the height and the value $1-a_{11}$ influences the width of the solution set $1 \gamma$. Similarly for the type $2 \gamma$, the value $1-a_{22}$ influences the height and the value $1-a_{21}$ influences the width of depicted solution set.
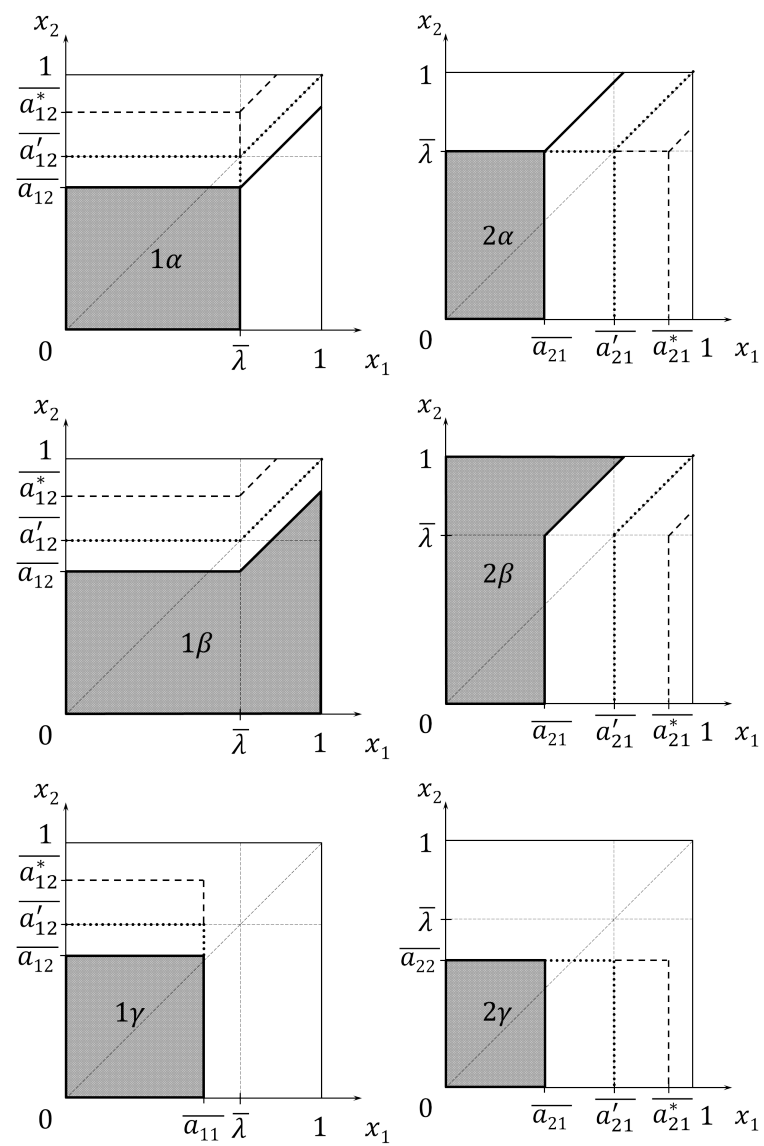

Figure 3: Types of solution sets for particular equations

The solution set of the eigenproblem is then an intersection of the solution sets of particular equations. Two numerical examples with graphical representation of the solution sets follow.

Example 3.1 Let us consider two matrices $A$ and $B$ and for both consider the same $\lambda=0.4$.

$$
A=\left(\begin{array}{cc}
0.3 & 0.8 \\
0.2 & 0.4
\end{array}\right) ; B=\left(\begin{array}{cc}
0.4 & 0.5 \\
0.2 & 0.4
\end{array}\right)
$$

To find the eigenspace associated with given lambda we will first determine the position of $\lambda$ and the diagonal entries of the matrices. For $\mathrm{A}$ it holds that $a_{11}<\lambda=a_{22}$. This means that the eigenspace is an intersection of the sets $1 \alpha$ and $2 \beta$. The fact that $a_{12}-\lambda>\lambda-a_{21}$ causes that the segment of solutions is shortened (left part of Figure 4).

Diagonal entries of $B$ are $a_{11}=a_{22}=\lambda$ and this leads to conclusion that the eigenspace of $B$ is an intersection of the sets $1 \beta$ and $2 \beta$. Note that the presence of the stripe of solutions (right part of Figure 4) is caused by the distances of the elements $a_{12}$ and $a_{21}$ from $\lambda$, specifically by inequality $a_{12}-$ $\lambda<\lambda-a_{21}$.
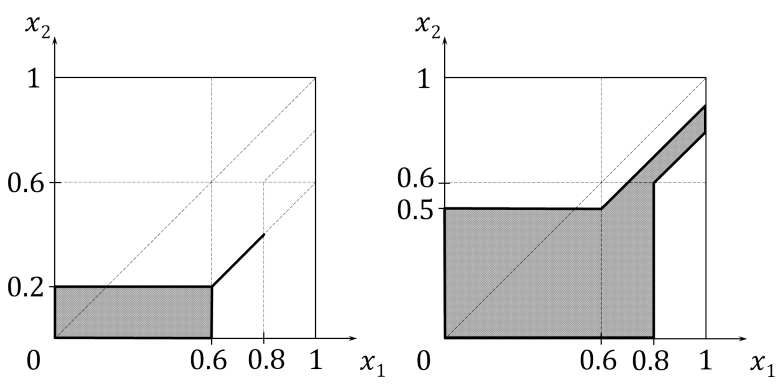

Figure 4: Solution sets for examples A (on the left) and $\mathrm{B}$ (on the right)

It can be seen from previous figures that the value $1-\lambda$ divides the coordinate system into four parts. The eigenvectors can be described by way of the partition of indices of the vector components (introduced in [3]) according to their value. For $x_{i} \leq 1-\lambda$ the index $i \in L$ and for $x_{i} \geq 1-\lambda$ include the index $i \in K$. Such partition is called $(K, L)$ partition. It is a partition of $\{1, \ldots, n\}$, that is, the subsets $K, L \subseteq\{1, \ldots, n\}$ such that $K \cup L=\{1, \ldots, n\}$ and $\bar{K} \cap L=\emptyset$, see Figure 5 . The letters in the circle indicate the membership of $i$ to the set according to the above written conditions, each of four parts of the whole set is then described by certain combination of subsets $K$ and $L$. The Eukasiewicz eigenvectors satisfying these conditions are called $(K, L)$-Łukasiewicz eigenvectors. On the other hand, if for some partition the $(K, L)$-Łukasiewicz eigenvectors exist, then we call such partition a "secure partition". For the given eigenspace in Figure 5 it holds that partitions $L=\{1,2\}, K=\emptyset ; L=\{2\}, K=\{1\}$ and $L=\emptyset, K=\{1,2\}$ are secure.

\section{The eigenspace for matrices of higher dimensions}

The complex algorithm for computation of the eigenspace using the $(K, L)$ partition is given in [3], see Algorithm 3.1. With the knowledge, which of the partitions are secure, we can compute the generators of the tropical convex hull for every secure partition. The solution of the eigenproblem is then an union of constituent solutions. 


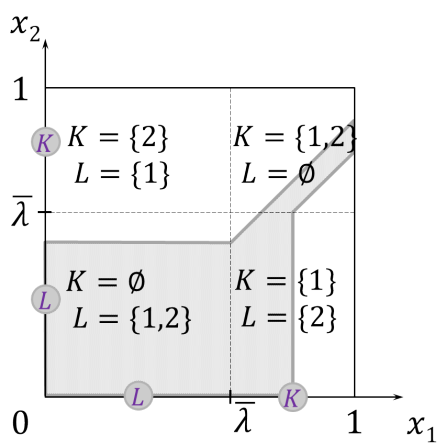

Figure 5: $(K, L)$ partition for two dimensions

To show the example for higher dimensions it is needful to define the "security".

We can distinguish three types of "security" [3]:

- for $\lambda>0$, a weighted digraph is called $\lambda$-secure if for each node of the graph and each walk $P$ issuing from that node holds $-\lambda+w(P) \leq 0$.

- the partition is called secure, if every walk in the weighted digraph of matrix $A^{(\lambda)}$, that starts in a node that belongs to $L$ and has all other nodes in $K$, has non-positive weight. We say that the partition $([n], \emptyset)$ is secure if corresponding directed graph of $A^{(\lambda)}$ is $\lambda$-secure, and that the partition $(\emptyset,[n])$ is secure. The notation $A^{(\lambda)}$ stands for the matrix with entries equal to $a_{i j}-\lambda$.

- node $i$ of a weighted digraph is called secure if the weight of every walk starting in $i$ is nonpositive.

The computation of the eigenspace for matrices of higher dimensions is shown on following threedimensional example.

Example 4.1 Consider the max-Łukasiewicz eigenproblem for $\lambda=0,7$ and

$$
A=\left(\begin{array}{lll}
0.6 & 0.8 & 0.4 \\
0.1 & 0.5 & 0.4 \\
0.9 & 0.6 & 0.5
\end{array}\right)
$$

Then we can write:

$$
A^{(\lambda)}=\left(\begin{array}{rrr}
-0.1 & 0.1 & -0.3 \\
-0.6 & -0.2 & -0.3 \\
0.2 & -0.1 & -0.2
\end{array}\right),
$$

It is easily seen that there are two positive paths in the directed graph of $A^{(\lambda)}$, see Figure 6 . For this simple three-dimensional example, according to definition of the security we are able to deduce, which of the partitions are secure. Let's start with the partition $L=\{2\}, K=\{1,3\}$. This partition is secure, because we can verify that every walk that starts in the node 2 and has all other nodes in $K$, that is, the walk can continue only between nodes 1 and 3 , has nonpositive weight.

To get further secure partition, we have to find such node in $K$ that is secure in $K$. This node, say

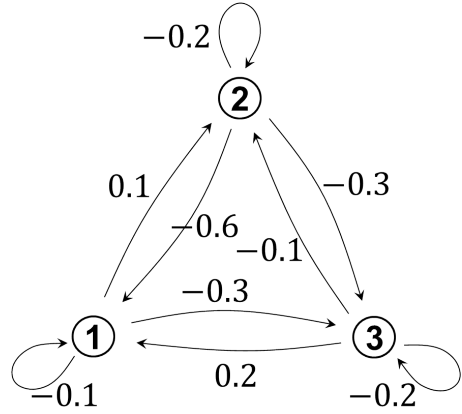

Figure 6: Weighted directed graph of $A^{(\lambda)}$

$k$, can be then shifted and the partition $(K-k, L+$ $k)$ is also a secure partition.

The node 3 is not secure in $K$, because of the walk $p=(3,1)$ with positive weight $w=0.2$. The node 1 is secure in $K$, because every walk realized from that node (that has all other nodes in $K$ ) has nonpositive weight. This node can be added to $L$ and we obtain next secure partition: $L=\{1,2\}, K=\{3\}$. According to definition, the partitions $L=\{1,2,3\}, K=\emptyset$ and $L=\emptyset, K=\{1,2,3\}$ are also secure.

To picture the $(K, L)$ partitions let's look at Figure 7 . The graphical representation is $3 \mathrm{D}$-cube divided (similarly as in two-dimensional example) by the value $\bar{\lambda}=1-\lambda$ on eight blocks.

The solution set of the eigenproblem is a union of tropical convex hulls for each of secure partitions, in this example, the union of solution sets of four blocks in the cube. We will focus on one of them, that one with the least $L$, the partition $L=\emptyset, K=$ $\{1,2,3\}$. It is the highlighted cube at the upper right corner in Figure 7.

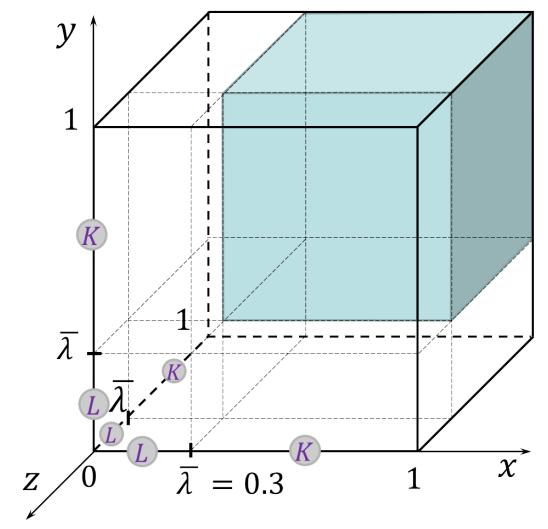

Figure 7: $(K, L)$ partition for three dimensions

Using Theorem 3.2. and Corollary 3.4. in [3], we compute the generators of tropical convex hull for the partition with the least $L$. We obtain two vectors, $u=\left(\begin{array}{lll}0.4 & 0.3 & 0.6\end{array}\right)$ and $w^{(1)}=\left(\begin{array}{lll}0.8 & 0.7 & 1\end{array}\right)$. These are the points depicted in Figure 8 . The point $w^{(1)}$ lies on the back side of the cube, whereas $u$ lies at the bottom. These two points are connected by the tropical segment. 


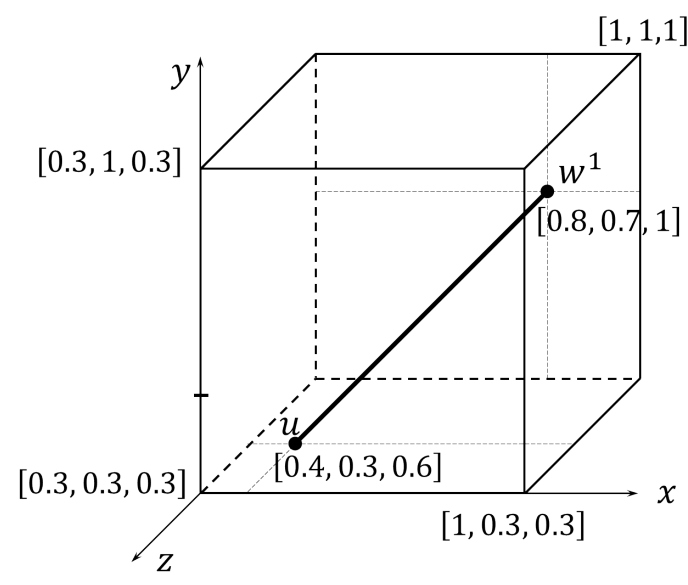

Figure 8: The tropical convex hull for the secure partition $L=\emptyset, K=\{1,2,3\}$

Similarly, we compute the generators for the next secure partitions (using Theorem 3.2. and Corollary 3.4.). The eigenspace for partition $L=\{2\}, K=$ $\{1,3\}$ is a tropical convex hull of vectors $u=$ $\left(\begin{array}{lll}0.3 & 0 & 0.5\end{array}\right), v^{(2)}=\left(\begin{array}{lll}0.4 & 0.3 & 0.6\end{array}\right), w^{(1)}=\left(\begin{array}{lll}0.4 & 0 & 0.6\end{array}\right)$. The generators of the eigenspace for the partition $L=\{1,2\}, K=\{3\}$ are vectors $u=\left(\begin{array}{lll}0 & 0 & 0.3\end{array}\right)$, $v^{(1)}=\left(\begin{array}{lll}0.3 & 0 & 0.5\end{array}\right), v^{(2)}=\left(\begin{array}{lll}0 & 0.2 & 0.3\end{array}\right)$. And, finally, the greatest background eigenvector for partition $L=\emptyset, K=\{1,2,3\}$ is $\left(\begin{array}{lll}0.1 & 0.2 & 0.3)\end{array}\right)$.

Observe, that the differences between each pair of coordinates of the generators depicted in Figure 8 equal to 0.4 , therefore the tropical segment is the segment parallel to the body diagonal of the universal set (every coordinate decreases by the same rate, notice the similarity to the graphical representation of the two-dimensional segment). As an example of not that simple segment we can take another two vectors, for example, two generators of the hull for the partition $L=\{2\}, K=\{1,3\}, u$ and $v^{(2)}$, depicted in Figure 9 (in fact figure represents the block located under the highlighted cube from Figure 7). These two points are also connected by the tropical segment. The point $v^{(2)}$ lies on the top of the block and the point $u$ lies right on the bottom left edge. Beginning from $v^{(2)}$, the segment is, again, parallel to the body diagonal of the universal set till it reaches the point $b=(0.30 .20 .5)$. From now, the only one coordinate, $y$, should decrease - the segment continues vertically down.

\section{Conclusions}

The study of eigenspace of a matrix in maxŁukasiewicz fuzzy algebra was introduced and the solution of the eigenproblem was provided on several examples together with the graphical representation of the solution sets.

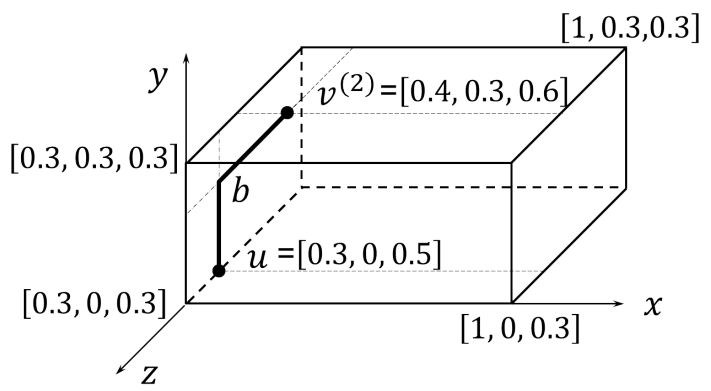

Figure 9: The tropical segment for three dimensions

\section{Acknowledgement}

The support of Specific Research project 2106/2015 at FIM UHK is kindly acknowledged.

\section{References}

[1] F. Bobillo and U. Straccia, Reasoning with the finitely many-valued Łukasiewicz fuzzy Description Logic, Information Sciences, Elsevier, 2011.

[2] S. Gottwald. A treatise on many-valued logics, Research Studies Press Baldock, 2001.

[3] M. Gavalec and Z. Němcová and S. Sergeev, Tropical linear algebra with the Łukasiewicz Tnorm, Fuzzy Sets and Systems, Elsevier, 2014.

[4] M. Gavalec and I. Rashid and R. Cimler, Eigenspace structure of a max-drast fuzzy matrix, Fuzzy Sets and Systems, 249:100-113, Elsevier, 2014.

[5] V. Nitica and I. Singer, The structure of maxplus hyperplanes, Linear Algebra and its Applications, 426:382-414, Elsevier, 2007.

[6] I. Rashid , M. Gavalec and S. Sergeev, Eigenspace of a three-dimensional maxŁukasiewicz fuzzy matrix, Kybernetika, 2012.

[7] I. Rashid and M. Gavalec and R. Cimler, Eigenspace structure of a max-prod fuzzy matrix, Fuzzy Sets and Systems, Elsevier, in prep.

[8] B. Schweizer and A. Sklar, Statistical metric spaces, Pacific J. Math, 10:313-335, 1960. 